\title{
Peptides as Versatile Platforms for Quantum Computing
}

\author{
Lorena E. Rosaleny, ${ }^{\dagger}$ Salvador Cardona-Serra, ${ }^{\dagger}$ Luis Escalera-Moreno, ${ }^{\dagger}$ José J. Baldoví, ${ }^{\dagger}$ \\ Violetta Golębiewska, ${ }^{\dagger}$ Karolina Wlazło, ${ }^{\dagger}$ Patricia Casino, ${ }^{\S}$ Helena Prima-García, ${ }^{*}{ }^{\dagger}$ \\ Alejandro Gaita-Ariño, ${ }^{*}{ }^{\dagger}$ and Eugenio Coronado ${ }^{\dagger}$

\begin{abstract}
${ }^{\dagger}$ Institut de Ciència Molecular, Universitat de València, Cat. José Beltrán 2, 46980 Paterna, Spain
${ }^{\ddagger}$ Max Planck Institute for the Structure and Dynamics of Matter, Luruper Chaussee 149, D-22761 Hamburg, Germany

${ }^{\S}$ Departamento de Bioquímica y Biología Molecular, ERI BioTecMed, Universitat de València, Dr. Moliner 50, 46100 Burjassot, Spain
\end{abstract}

Supporting Information

\begin{abstract}
The pursuit of novel functional building blocks for the emerging field of quantum computing is one of the most appealing topics in the context of quantum technologies. Herein we showcase the urgency of introducing peptides as versatile platforms for quantum computing. In particular, we focus on lanthanide-binding tags, originally developed for the study of protein structure. We use pulsed electronic paramagnetic resonance to demonstrate quantum coherent oscillations in both neodymium and gadolinium peptidic qubits. Calculations based on density functional theory followed by a ligand field analysis indicate the possibility of influencing the nature of the spin qubit states by means of controlled changes in the peptidic sequence. We conclude with an overview of the challenges and opportunities opened by this interdisciplinary field.
\end{abstract}

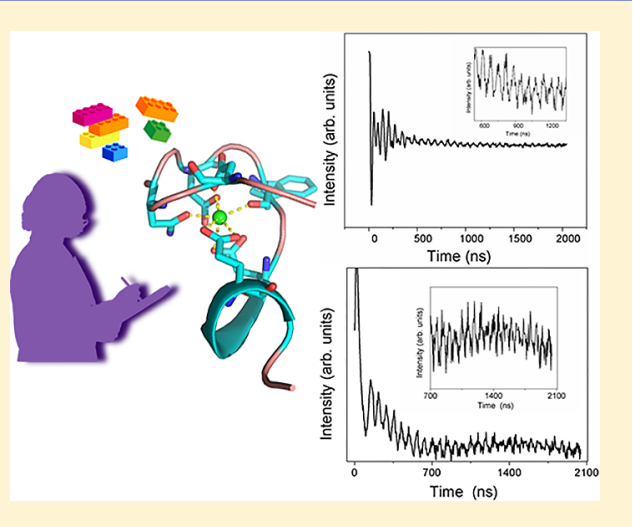

uantum computing is an emerging scientific field that holds the promise of dramatically enhancing the current capabilities of information and communication technologies. In this framework, one of the most encouraging potential applications of magnetic molecules is their integration as "smart" building blocks in quantum computers. ${ }^{1,2}$ The exploration of biomolecules as versatile platforms within this paradigm opens the door to a detailed engineering of each component thanks to techniques developed in molecular biology, including recombinant protein technology ${ }^{3}$ or CRISPR-CAS9. ${ }^{4}$ These techniques enable the fabrication of relatively inexpensive, on-demand peptide sequences and the modification of existing proteins in a standard way, facilitating tailored molecular preparation. The implementation of spincoherent phenomena in spin-labeled proteins or polypeptides via artificial processes that imitate quantum biology ${ }^{5-7}$ offers a promising avenue with far-reaching consequences in nanoscience and nanotechnology. Thus it is urgent to verify if these magnetic building blocks can display spin coherence and can be structurally stable in different environments. On the one hand, this would add metallopeptides to the race, placing them in direct competition with purely chemical strategies previously reported for the organization of spin qubits. ${ }^{8,9}$ On the other hand, they would be in an interesting position to play a supporting role for the mixed chemical-physical approach for scaling of qubits, such as the coupling of individual multiqubit molecules with superconducting transmission lines. ${ }^{1,2,10}$ The first step toward this direction considers very simple radicallabeled peptides forming a small molecular spin quantum network that can be controlled via a nitrogen-vacancy center in diamond; ${ }^{11}$ however, stability problems were reported in this case. The controlled preparation of stable biomolecules with distinct spin ground doublets would be the next natural step of this approach.

Herein we propose to combine peptides with lanthanide ions, which are well established as a spin component with potential for quantum technologies. ${ }^{2,12}$ Among interesting results demonstrated recently with lanthanide molecular complexes, we need to highlight magnetic hysteresis at 60 $\mathrm{K},{ }^{13}$ implementation of clock transitions, ${ }^{14}$ and the integration of a three-qubit quantum processor in a single ion. ${ }^{1}$ In this work, we report our advances toward the design of coherent, scalable, peptidic spin qubits based on lanthanide ions. The path toward this goal starts with the preparation and characterization of a single peptidic spin qubit, including coherent operations, and progresses into the demonstration of a certain control over the coordination environment of each qubit. The manuscript will commence by describing lanthanide binding tags, ${ }^{15}$ which are the particular metallopeptides on which we will focus as a case study, and demonstrating that they can be considered autonomously folding domains, meaning they are structurally robust toward perturbations in their environment. ${ }^{16}$ We will proceed to show their potential as

Received: June 11, 2018

Accepted: July 25, 2018

Published: July 25, 2018 
spin qubits in terms of quantum coherence and investigate the possibility of influencing their magnetic properties by a careful control of the peptidic sequence. Finally, we will comment on the procedure of property optimization via peptidic screening.

Choice for Biomolecular Spin Qubit. Employing biomolecules as hardware is a general strategy. It can conceivably be implemented in many different ways, with candidates including all metalloproteins, as well as radical-tagged peptides. ${ }^{11}$ Equivalently, DNA self-assembly ${ }^{17}$ could be used for this same goal. As a proof of concept, in this work, we need to focus on a particular case, both in terms of the magnetic ion and in terms of the biomolecule. We chose a metallopeptide that some of us are simultaneously exploring as a component for biomolecular spintronics. ${ }^{18}$

In particular, in this work, we used electronic paramagnetic resonance $(\mathrm{EPR})$ to probe trivalent lanthanide ions $\mathrm{Ln}=\mathrm{Nd}$, Gd, Tb, Dy, Ho, Er, Tm, Yb. As biomolecular component, we chose a short peptide sequence ( 17 amino acids) known as lanthanide binding tag (see Figure 1). ${ }^{19}$ These were originally (a)

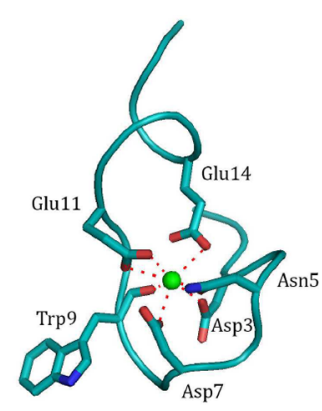

(b)

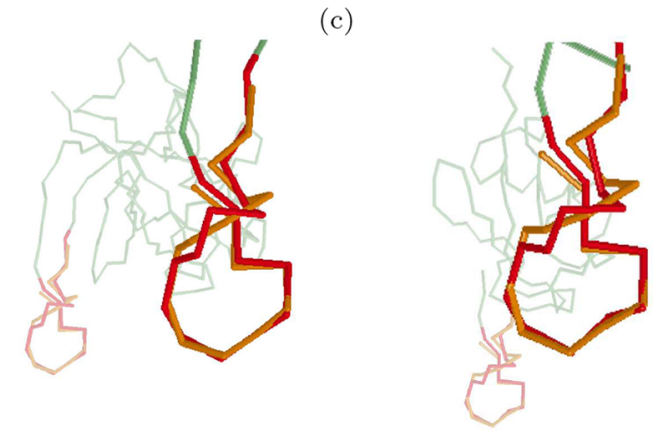

Figure 1. (a) $\mathrm{C}_{\alpha}$ trace from peptide $1 \mathrm{TJB}$ with metal-coordinating residues represented as sticks, terbium as a green sphere, and metal-O bonds as red dashes. (b,c) Alignment and superposition, calculated using TopMatch $^{20}$ of 1TJB (in orange) with similar sequences either fused with a ubiquitin (b) or inserted in the middle S-loop of an interleukin-1- $\beta$ (c), with the matching region highlighted in red. Very similar foldings (highlighted, front) in completely different proteins (transparent, back).

optimized by Imperiali et al. via the exploration of a series of peptide libraries, starting from calcium-binding motifs of proteins, with the combined goals of fine-tuning the selectivity of binding to lanthanide ions and presenting photoluminescence. $^{21}$ As any short peptide, these molecules can easily be genetically encoded, and thus they are used as luminescent spin tags in controlled protein positions. ${ }^{15}$

The most standard lanthanide binding tag, employing the one-letter code for amino acids, is YIDTNNDGWYEGDELLA (from now on: LBT), and several lanthanide-binding peptides are closely related to it (see Table S1), and thus we employed it as a test case. In this work, all LnLBT samples were prepared from commercially acquired LBT.

Structural and Quantum Robustness. Let us start by demonstrating the autonomous folding of LBT, its structural robustness. In general, the folding of a sequence of amino acids depends on the details of its sequence and its environment, and thus a modified peptide can, in principle, fold in different ways. To determine whether this problem can affect LBT, we employed a combination of TopMatch and SHAPE codes. ${ }^{22}$ TopMatch (Figure $1 \mathrm{~b}, \mathrm{c}$ ) demonstrates that the folding is robust and consistent with vastly different environments. SHAPE employs a different theoretical approach to obtain the same result: All known instances in this family of metallopeptides fold in the same way. (See Section S1 of the Supporting Information for details.)

With respect to quantum robustness, peptides have a high number of vibrational degrees of freedom and a high number of nuclear spins (from $I=1 / 2{ }^{1} \mathrm{H}$ atoms), so one would naïvely expect short relaxation times compared with regular coordination compounds. However, a previous theoretical study by some of us considering the ${ }^{1} \mathrm{H}$ nuclear spin bath as decoherence source estimated an upper limit of the decoherence times above tens of microseconds in several members of the LnLBT series, ${ }^{23}$ and indeed experimental results in a different context report spin echoes in these kind of complexes. $^{24}$

In this work, we performed a more exhaustive EPR characterization, including both continuous-wave and pulsed EPR. In the preliminary continuous-wave EPR exploration, we only obtained signal for GdLBT and NdLBT. (For details, see the Supporting Information Section S2.) This allowed an estimate of their spin energy levels and wave functions. (For details, see the Supporting Information Section S3.) For these two derivatives we also found a signal in the pulsed experiments. The relaxation times were found to be at least comparable to those typical for Ln complexes, ${ }^{1}$ with $T_{1} \simeq 360$ $\mu \mathrm{s}, x=0.54$ and $T_{\mathrm{m}} \simeq 2.5 \mu \mathrm{s}, x=1.6$ in the case of GdLBT (see Figure S2.4). Note that this is also consistent with recent DEER measurements in other Gd-complexing LBT-tagged proteins ${ }^{24}$ where $T_{\mathrm{m}} \simeq 2 \mu$ s had also been measured. In the case of NdLBT, the relaxation times are noticeably shorter, $T_{1}$ $\simeq 8 \mu \mathrm{s}, x=0.39$ and $T_{\mathrm{m}} \simeq 0.6 \mu \mathrm{s}$ and $x=0.74$.

The contribution of the proton spin bath was estimated to be moderate, providing a ceiling for the coherence time on the order of $T_{\mathrm{m}}<300 \mu \mathrm{s}$, meaning it is not the limiting factor in this case; see the Supporting Information Section S4.

One needs to note here that sufficiently long $T_{1}$ and $T_{\mathrm{m}}$ times are a necessity but not a guarantee for a useful qubit. These parameters only quantify the survival of quantum information that is not actually being manipulated, which is not a practical setup. Coherent oscillations are much more interesting: They are the back-and-forth oscillations between two quantum states, like clock cycles. In very noisy systems, such as most solid-state systems, quantum coherence is lost on such a short time scale that coherent oscillations are impossible: Detecting coherent oscillations for at least a few cycles is considered a sine qua non condition for the use of a particular hardware for quantum applications. We studied coherent oscillations at the Hartmann-Hahn conditions for best performance, ${ }^{25}$ both for GdLBT and for NdLBT, obtaining several Larmor oscillations (Figure 2). Note that under these conditions the relation between oscillation frequency $\Omega_{\mathrm{R}}$ and $B_{1}$ is outside the linear regime characterizing 

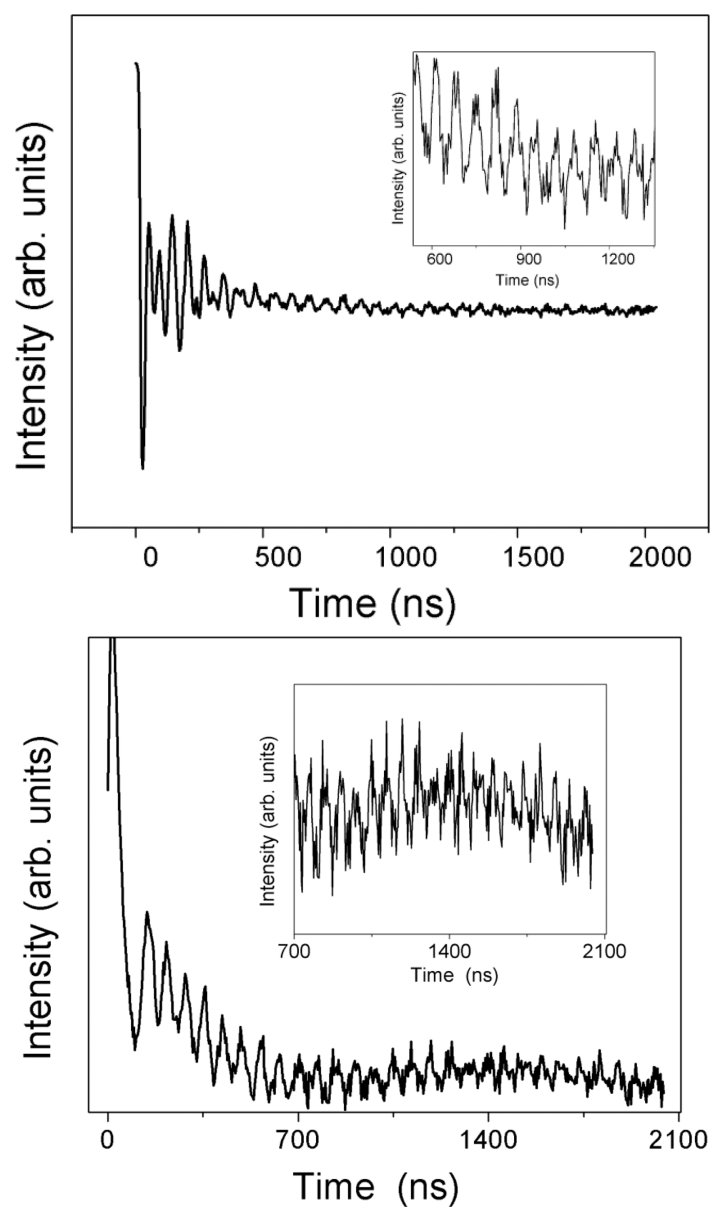

Figure 2. Coherent oscillations at $4 \mathrm{~K}$ in GdLBT (top) and NdLBT (bottom) under the Hartmann-Hahn condition. ${ }^{25}$ Inset: Zoom at the regime with negligible decay.
Rabi oscillations (Figure S2.1b). The current $T_{\mathrm{m}}$ values and this number of oscillations, establish that peptidic qubits are already in the same range of coherence as the majority of nonoptimized coordination chemistry complexes.

Tunability of the Qubit by Choice of the Peptidic Sequence. We have established the sufficient coherence of biomolecular spin qubits, but the motivation for the use of biomolecular qubits is not a high coherence but rather the customization of the qubit environment. Because of the robustness of the folding in these peptides, as discussed above, it has been shown that it is possible to substitute an asparagine (carboxamide) by an aspartate (carboxylate) with no change in the structure. Indeed, in LnLBT, there are three monocoordinating positions (Asp3, Asn5, and Asp7 in Figure 1c) that can, in principle, be provided by either aspartate or asparagine residues, giving rise to up to eight combinations. For our purposes, this means that it is possible to make controlled changes in the coordination environment, altering the distribution of the charges but maintaining the locations of the ligands. As a further independent verification of this phenomenon, we employed the SIESTA software package ${ }^{26}$ to complete these crystallographic comparisons with a series of density functional theory (DFT) structural relaxations of peptides, comparing the original LBT sequence YIDTNNDGWYEGDELLA (DND), with two minor modifications in the sequence that preserve the total charge: YINTDNDGWYEGDELLA (NDD) and YIDTDNNGWYEGDELLA (DDN). In all three cases, the atomic displacements were minimal (see Figure S5.1).

Having established the robustness of the peptide folding against these particular amino acid permutations, we performed a theoretical study on the possibility of influencing the nature of the ground doublet, in particular, the composition in terms of $M_{I}$ components of its wave function by means of controlled changes in the peptidic sequence. We limited ourselves here to three sequences with equal charge,
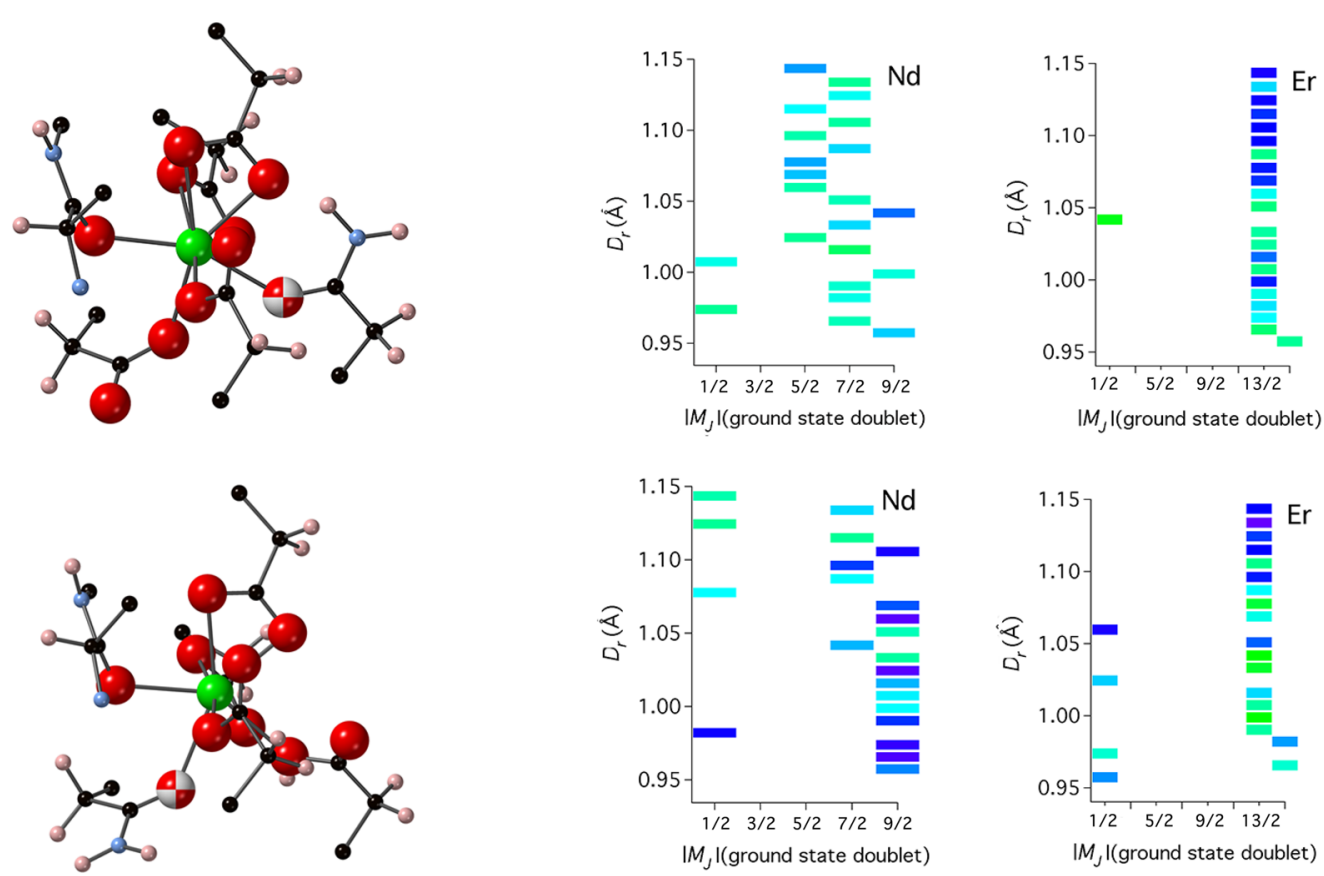

$\left|M_{j}\right|$ (ground state doublet)

$|M$,$| (ground state doublet)$

Figure 3. Alteration of the peptidic sequence affects the charge distribution and thus the ground doublet. Top and bottom rows refer to DND and NDD peptidic sequences, respectively. Left: coordination environment, Ln (green), O ( red), N (blue), C (black), and H (pink), highlighting the position of the asparagine (carboxamide) oxygen; right: estimation of the ground doublet employing the REC model for Nd and for Er. 
DND, NDD, and DDN, to minimize the number of variables we are affecting simultaneously.

Starting from the DFT-relaxed sequences, we employed the SIMPRE software within the radial effective charge (REC) model, ${ }^{27-29}$ which allows us to reproduce covalency effects by using an effective charge and an effective radial displacement $D_{\mathrm{r}}$. (See Supporting Information Section S5 for details.) As an example of the effect in the coordination sphere of this change in the peptidic sequence, see Figure 3.

We explored LnLBT* where Ln $=(\mathrm{Nd}, \mathrm{Tb}, \mathrm{Dy}, \mathrm{Ho}, \mathrm{Er}, \mathrm{Tm}$, $\mathrm{Yb})$ and $\mathrm{LBT}^{*}=($ DND, NDD, DDN$)$ with a sampling of REC parameters. We found that, in general, a mere exchange between asparagine and aspartate is not expected to alter the magnetic properties of the ground doublet. For example, Dy complexes with these slight variations of the LBT sequence are, for a wide range of REC parameters, consistently expected to present a $M_{J}= \pm 15 / 2$ ground doublet.

In contrast, our calculations indicate that obtaining a different ground doublet spin through a minimal and controlled change in the peptidic sequence seems entirely possible for NdLBT*. As can be seen in Figure 3, in the DND $\mathrm{Nd}$ complex, the models tend to predict a rather mixed ground doublet, with the dominant term being either $M_{I}= \pm 7 / 2$ or $M_{I}$ $= \pm 5 / 2$, whereas for NDD Nd complex, the models consistently predict a less mixed $M_{I}= \pm 9 / 2$. In the case of Er, which we use here as a typical example for comparison, the models consistently predict that the dominant term is $M_{J}=$ $\pm 13 / 2$, independently of the peptidic sequence. The results are compatible with the fits of the CW-EPR spectrum of DND, which result in an easy-axis anisotropy. (See Supporting Information Section S3 for details.)

A further key advantage of peptides as qubits is property optimization via the combinatorial screening of peptide libraries. (See more details in the Supporting Information Section S6.) In principle, it should be possible to minimize the coupling between the spin qubit and molecular vibrations by finding the sequence of amino acids where low-energy vibrations do not alter the coordination environment of the magnetic metal. ${ }^{30,31}$

Peptides known as lanthanide binding tags were developed in the field of biochemistry for the study of protein structure and dynamics. Herein we have expanded their applications and shown that they can be used as spin tags to open a promising playground as molecular spin qubits. Among the advantages of employing metallopeptides as spin qubits are the ease of preparing new structures and the possibility to customize their properties and organize them in space using techniques of molecular biology. Furthermore, it should be possible to optimize quantum coherence via peptidic library screening. Homogeneous orientation of these anisotropic spins can be achieved in ensemble measurements by crystallization, which is admittedly challenging. Indeed it was not achieved in this case, even after applying a wide array of crystallization conditions. (See the Supporting Information Section S7.) In the case of future single-molecular experiments, it is a feasible task to tag a protein of known structure in a known position with our qubits, and this would serve to achieve the desired orientation of the spins. ${ }^{32}$ This work proves that metallopeptides open different avenues for quantum computing that are closed to conventional coordination chemistry.

\section{ASSOCIATED CONTENT}

\section{Supporting Information}

The Supporting Information is available free of charge on the ACS Publications website at DOI: 10.1021/acs.jpclett.8b01813.

Additional information on several experimental and theoretical details and also on future perspectives, a spectroscopic characterization, additional CW EPR and pulsed EPR experimental characterization, considerations related to folding patterns (SHAPE), and a summary of the crystallization efforts (PDF)

\section{AUTHOR INFORMATION}

\section{Corresponding Authors}

*H.P.-G.: E-mail: helena.prima@uv.es.

*A.G.-A.: E-mail: alejandro.gaita@uv.es.

ORCID

Lorena E. Rosaleny: 0000-0002-8481-7303

Salvador Cardona-Serra: 0000-0002-5328-7047

Alejandro Gaita-Ariño: 0000-0002-1600-8627

Notes

The authors declare no competing financial interest.

\section{ACKNOWLEDGMENTS}

The present work has been funded by the EU (ERC Consolidator Grant 647301 DECRESIM, ERC Advanced Grant 788222 Mol-2D, and COST 15128 Molecular Spintronics Project), the Spanish MINECO (grants MAT2017-89528 and CTQ2017-89993 cofinanced by FEDER and Excellence Unit María de Maeztu MDM-20150538), and the Generalitat Valenciana (Prometeo Program of Excellence). A.G.-A. acknowledges funding by the MINECO (Ramón y Cajal Program), L.E.-M. thanks the Generalitat Valenciana for a VALi+d predoctoral grant. J.J.B. thanks the EU for a Marie Curie Fellowship (H2020-MSCA-IF-2016751047). S.C.-S. thanks the Spanish MINECO for a Juan de la Cierva Incorporación postdoctoral Fellowship. We thank Samuel Mañas-Valero and José V. Usagre for their assistance in the preparation of the EPR samples.

\section{REFERENCES}

(1) Jenkins, M. D.; Duan, Y.; Diosdado, B.; García-Ripoll, J. J.; Gaita-Ariño, A.; Giménez-Saiz, C.; Alonso, P. J.; Coronado, E.; Luis, F. Coherent Manipulation of Three-Qubit States in a Molecular Single-Ion Magnet. Phys. Rev. B: Condens. Matter Mater. Phys. 2017, 95, 064423.

(2) Gaita-Ariño, A.; Luis, F.; Hill, S.; Coronado, E. Mononuclear Rare-Earth Nanomagnets as a Source of Spin-Qubits Obtained by Chemical Design. Nat. Chem. 2018, under review.

(3) Wingfield, P. T. Overview of the Purification of Recombinant Proteins. Curr. Protoc. Protein Sci. 2015, 80, 6.1.1-6.1.35.

(4) Sternberg, S. H.; Doudna, J. A. Expanding the Biologists Toolkit with CRISPR-Cas9. Mol. Cell 2015, 58, 568-574.

(5) Lambert, N.; Chen, Y.-N.; Cheng, Y.-C.; Li, C.-M.; Chen, G.-Y.; Nori, F. Quantum Biology. Nat. Phys. 2013, 9, 10-18.

(6) Cai, J.; Plenio, M. B. Chemical Compass Model for Avian Magnetoreception as a Quantum Coherent Device. Phys. Rev. Lett. 2013, 111, 230503.

(7) Scholes, G. D.; Fleming, G. R.; Chen, L. X.; Aspuru-Guzik, A.; Buchleitner, A.; Coker, D. F.; Engel, G. S.; van Grondelle, R.; Ishizaki, A.; Jonas, D. M.; et al. Using Coherence to Enhance Function in Chemical and Biophysical Systems. Nature 2017, 543, 647-656. 
(8) Ferrando-Soria, J.; Moreno Pineda, E.; Chiesa, A.; Fernandez, A.; Magee, S. A.; Carretta, S.; Santini, P.; Vitorica-Yrezabal, I. J.; Tuna, F.; Timco, G. A.; et al. A Modular Design of Molecular Qubits to Implement Universal Quantum Gates. Nat. Commun. 2016, 7, 11377.

(9) Boulon, M. E.; Fernandez, A.; Moreno-Pineda, E.; Chilton, N. F.; Timco, G.; Fielding, A. F.; Winpenny, R. E. P. Measuring Spin $\cdots$ Spin Interactions between Heterospins in a Hybrid [2]Rotaxane. Angew. Chem., Int. Ed. 2017, 56, 3876.

(10) Jenkins, M. D.; Zueco, D.; Roubeau, O.; Aromí, G.; Majer, J.; Luis, F. A. Scalable Architecture for Quantum Computation with Molecular Nanomagnets. Dalton Trans. 2016, 45, 16682-16693.

(11) Schlipf, L.; Oeckinghaus, T.; Xu, K.; Dasari, D. B. R.; Zappe, A.; de Oliveira, F. F.; Kern, B.; Azarkh, M.; Drescher, M.; Ternes, M.; et al. A Molecular Quantum Spin Network Controlled by a Single Qubit. Science Advances 2017, 3, e1701116.

(12) McAdams, S. G.; Ariciu, A.-M.; Kostopoulos, A. K.; Walsh, J. P. S.; Tuna, F. Molecular Single-Ion Magnets Based on Lanthanides and Actinides: Design Considerations and New Advances in the Context of Quantum Technologies. Coord. Chem. Rev. 2017, 346, 216.

(13) Goodwin, C. A. P.; Ortu, F.; Reta, D.; Chilton, N. F.; Mills, D. P. Molecular Magnetic Hysteresis at $60 \mathrm{~K}$ in Dysprosocenium. Nature 2017, 548, 439-442.

(14) Shiddiq, M.; Komijani, D.; Duan, Y.; Gaita-Ariño, A.; Coronado, E.; Hill, S. Enhancing Coherence in Molecular Spin Qubits Via Atomic Clock Transitions. Nature 2016, 531, 348-351.

(15) Allen, K. N.; Imperiali, B. Lanthanide-Tagged Proteins - an Illuminating Partnership. Curr. Opin. Chem. Biol. 2010, 14, 247-254.

(16) Schaeffer, R. D.; Daggett, V. Protein Folds and Protein Folding. Protein Eng., Des. Sel. 2011, 24, 11-19.

(17) Zhang, F.; Yan, H. DNA Self-Assembly Scaled Up. Nature 2017, 552, 34.

(18) Cardona-Serra, S.; Torres Cavanillas, R.; Escorcia-Ariza, G.; Giménez-Santamarina, S.; Sessolo, M.; Galbiati, M.; Tatay, S.; Rosaleny, L. E.; Forment-Aliaga, A.; Gaita-Ariño, A. A LanthanoidComplexing Peptide as a Single-Component Spin Valve. Inorg. Chem. 2018, under review.

(19) Franz, K. J.; Nitz, M.; Imperiali, B. Lanthanide-Binding Tags as Versatile Protein Coexpression Probes. ChemBioChem 2003, 4, 265271.

(20) Sippl, M. J.; Wiederstein, M. Detection of Spatial Correlations in Protein Structures and Molecular Complexes. Structure 2012, 20, $718-728$.

(21) Nitz, M.; Franz, K. J.; Maglathlin, R. L.; Imperiali, B. A Powerful Combinatorial Screen to Identify High-Affinity Terbium(III)-Binding Peptides. ChemBioChem 2003, 4, 272-276.

(22) Alvarez, S.; Alemany, P.; Casanova, D.; Cirera, J.; Llunell, M.; Avnir, D. Shape Maps and Polyhedral Interconversion Paths in Transition Metal Chemistry. Coord. Chem. Rev. 2005, 249, 16931708.

(23) Rosaleny, L. E.; Gaita-Ariño, A. Theoretical Evaluation of Lanthanide Binding Tags as Biomolecular Handles for the Organization of Single Ion Magnets and Spin Qubits. Inorg. Chem. Front. 2016, 3, 61-66.

(24) Barthelmes, D.; Gränz, M.; Barthelmes, K.; Allen, K. N.; Imperiali, B.; Prisner, T.; Schwalbe, H. Encoded Loop-LanthanideBinding Tags for Long-Range Distance Measurements in Proteins by NMR and EPR Spectroscopy. J. Biomol. NMR 2015, 63, 275-282.

(25) Baldoví, J. J.; Cardona-Serra, S.; Clemente-Juan, J. M.; Coronado, E.; Gaita-Ariño, A.; Prima-García, H. Coherent Manipulation of Spin Qubits Based on Polyoxometalates: the Case of the Single Ion Magnet $\left[\mathrm{GdW}_{30} \mathrm{P}_{5} \mathrm{O}_{110}\right]^{14-}$. Chem. Commun. 2013, 49, $8922-8924$.

(26) Soler, J. M.; Artacho, E.; Gale, J. D.; García, A.; Junquera, J.; Ordejón, P.; Sánchez-Portal, D. The SIESTA Method for $A b$ Initio Order-N Materials Simulation. J. Phys.: Condens. Matter 2002, 14, 2745-2779.

(27) Baldoví, J. J.; Cardona-Serra, S.; Clemente-Juan, J. M.; Coronado, E.; Gaita-Ariño, A.; Palii, A. SIMPRE: A Software Package to Calculate Crystal Field Parameters, Energy Levels, and Magnetic
Properties on Mononuclear Lanthanoid Complexes Based on Charge Distributions. J. Comput. Chem. 2013, 34, 1961-1967.

(28) Baldoví, J. J.; Borrás-Almenar, J. J.; Clemente-Juan, J. M.; Coronado, E.; Gaita-Ariño, A. Modeling the Properties of Lanthanoid Single-Ion Magnets Using an Effective Point-Charge Approach. Dalton Trans. 2012, 41, 13705-13710.

(29) Baldoví, J. J.; Gaita-Ariño, A.; Coronado, E. Modeling the Magnetic Properties of Lanthanide Complexes: Relationship of the REC Parameters with Pauling Electronegativity and Coordination Number. Dalton Trans. 2015, 44, 12535-12538.

(30) Escalera-Moreno, L.; Suaud, N.; Gaita-Ariño, A.; Coronado, E. Determining Key Local Vibrations in the Relaxation of Molecular Spin Qubits and Single-Molecule Magnets. J. Phys. Chem. Lett. 2017, $8,1695-1700$.

(31) Escalera-Moreno, L.; Baldoví, J. J.; Gaita-Ariño, A.; Coronado, E. Spin States, Vibrations and Spin Relaxation in Molecular Nanomagnets and Spin Qubits: a Critical Perspective. Chem. Sci. 2018, 9, 3265-3275.

(32) Gaita-Ariño, A.; Prima-García, H.; Cardona-Serra, S.; EscaleraMoreno, L.; Rosaleny, L. E.; Baldoví, J. J. Coherence and Organisation in Lanthanoid Complexes: from Single Ion Magnets to Spin Qubits. Inorg. Chem. Front. 2016, 3, 568-577. 